\title{
Unilateral appearance of a chondro-epitrochlearis muscle- a case report
}

\section{P. Jaijesh}

Department of Anatomy, Melaka Manipal Medical College, Manipal -576104, Karnataka, India

Address for correspondence: Dr. Paval Jaijesh, Melaka Manipal Medical College, Manipal - 576104, Karnataka, India.

E-mail: jaijesh@yahoo.co.in

\section{ABSTRACT}

Variant muscle slips from pectoralis major muscle are rare. Among these, the muscle chondroepitrochlearis is a very rare muscular anomaly. Here, in this report, we describe a similar muscle which had an origin from the lower ribs along with the lower fibres of the pectoralis major muscle, arched across the axilla, and then inserted to the medial epicondyle of humerus. In this report we present a review of literature on this muscle. We also discuss the clinical significance of this muscle since the knowledge of this muscle is important in the differential diagnosis of ulnar nerve entrapment.

\section{KEY WORDS}

Chondro-epitrochlearis, Pectoralis major muscle, Ulnar artery 


\section{INTRODUCTION}

hondro-epitrochlearis is a very rare muscular anomaly. This is the first instance of this anomalous muscle we have encountered over a period of 25 years in our dissection hall. Bergman ${ }^{1}$ describes this anomaly as a muscular slip which arises from one or more ribs, crosses the axilla and inserts into the medial intermuscular septum or medial epicondyle of the humerus. According to $\mathrm{Wood}^{2}$ this muscle should be described as 'epigastric slips' represented by the detached slips from the inferior border of pectoralis major that insert on the humerus on the deep surface of its own tendon of insertion. Landry et $a l^{3}$ have described the phylogenetic significance of such a variant muscle and Spinner et al. ${ }^{4}$ have emphasized its clinical relevance.

\section{CASE REPORT}

During the routine dissection for the medical students we found this muscle unilaterally in a male cadaver. The muscle had an origin from the sixth costal cartilage near the costochondral junction. The muscle continued along the inferolateral border of the pectoralis major muscle. Upon reaching the axilla, this muscle arched towards the arm, formed a narrow tendon, crossed brachial artery and inserted to the superior surface of the medial epicondyle of the humerus. In its course towards the medial epicondyle, the muscle was found plastered to the medial head of the triceps brachii muscle. The ulnar nerve was found between the tendon of this muscle and medial head of triceps muscle. [Figure 1, 2]

\section{DISCUSSION}

The muscle described in the present report is similar to that described by Perrin. ${ }^{5} \mathrm{He}$ also identified a muscle which terminated opposite the middle of the anterior aspect of the arm, which had descended from the capsule of the shoulder joint anterior to the tendon of the long head of biceps brachii muscle. Bregman et al. ${ }^{1}$ and Anson ${ }^{6}$ found a similar muscle in 12 to $20 \%$ bodies. But considering the current literature, this kind of high prevalence of this anomalous muscle is not well established.

Although the tendinous insertion of this muscle is the common one, Bryce ${ }^{7}$ reported a fleshy insertion of this muscle in the medial intermuscular septum 2 inches above the medial epicondyle. Macalister ${ }^{8}$ in his work on human muscular variations makes a reference to a digastric chondro-epitrochlearis.

Nerve supply of this muscle is mostly derived from the pectoral branches of the brachial plexus. Bryce ${ }^{7}$ and laundry ${ }^{3}$ reported about the nerve supply of this muscle which derived from an anterior division of the brachial plexus and believe that this may be due to its embryological origin from the ventral muscle mass. A dual innervation was reported by Spinner et $a l,{ }^{4}$ one from the Y- junction of the medial and lateral roots of the median nerve, and the other from the branch of the medial pectoral nerve. In the present case the muscle is innervated by the medial pectoral nerve.

Chiba et $a l^{9}$ suggested that the chondro-epitrochlearis is

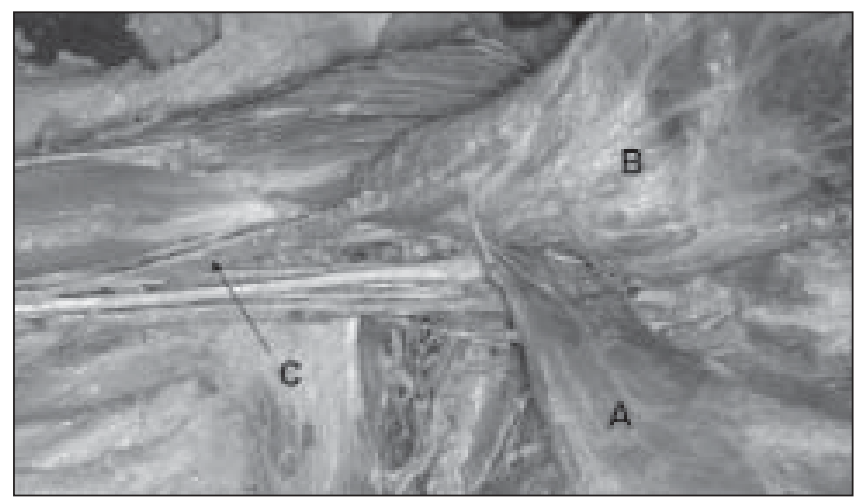

Figure 1: A-Pectoralis minor B-Pectoralis major (reflected) C-Chondroepitrochlearis. Figure showing the origin of chondro-epitrochlearis muscle

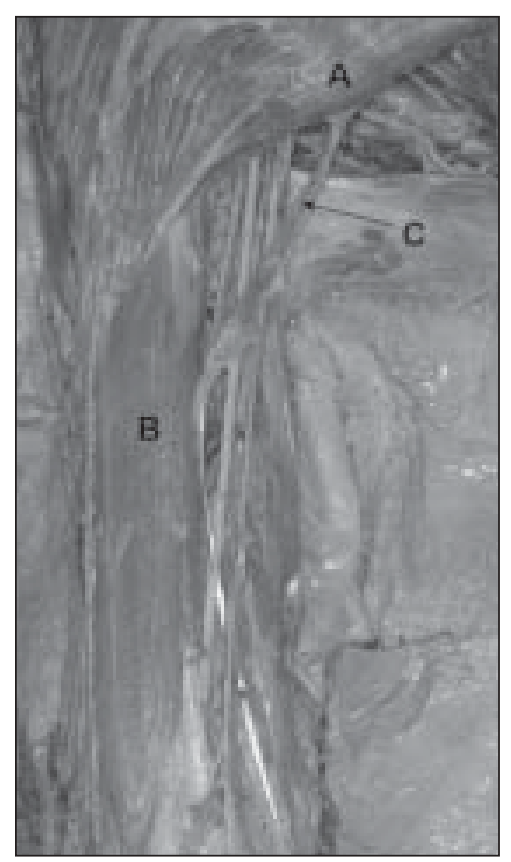

Figure 2: A-Pectoralis major B-Biceps brachii C-Chondro-epitrochlearis Figure showing the complete course of the chondro-epitrochlearis muscle 
almost always associated with the axillary arch muscle, which itself occurs in 7 to $13 \%$ of the population. Voto and Weine ${ }^{10}$ reported a clinical case of an infant with a contracture of the chondro-epitrochlearis in whom the axillary arch muscle was absent.

According to Landry ${ }^{3}$ and Bergman ${ }^{11}$ the presence of an anomalous chondro-epitrochlearis is invariably associated with the absence of the usual twist of tendon of insertion of pectoralis major. But in the present case, pectoralis muscle had the usual twisted insertion and also the axillary arch muscle was absent.

Landry ${ }^{3}$ reported that chondro-epitrochlearis is an atavistic anomaly which demonstrates the distal extent of the insertion in ancestral forms. The chondro-epitrochlearis muscle has been found at autopsy in infants with chromosomal defects, namely, trisomy 13 with a D/D translocation and trisomy $18 .{ }^{12}$ In the present case, the ulnar nerve was found entrapped between the tendon of chondro-epitrochlearis and the medial head of triceps brachii. This situation of ulnar nerve entrapment will be mimicking the manifestations of cubital tunnel syndrome or the situation where the struther's ligament is involved.

To conclude, this muscle anomaly is clinically important for its potential to cause cosmetic defects, to restrict abduction of the arm and also for the differential diagnosis of the compressive neuropathy from the ulnar nerve entrapment.

\section{REFERENCES}

1. Bergman RA, Thompson SA, Afifi AK, Saadeh FA. Compendium of Human Anatomic Variation. Munchen, Baltimore: Urban and Schwarzenberg.1988

2. Wood J. Variations in human myology observed during the winter session of 1867-68 at King's College, London. Proceedings of the Royal Society 1868;17:483-525.

3. Landry SO. The phylogenetic significance of the chondroepitrochlearis muscle and its accompanying pectoral abnormalities. Journal of Anatomy 1958;92:57-64.

4. Spinner RJ, Carmichael SW, Spinner M. Infraclavicular ulnar nerve entrapment due to a chondro-epitrochlearis muscle. Journal of Hand Surgery 1991;16B:315-7.

5. Perrin JB. Notes on some variations of the pectoralis major, with its associate muscles. Journal of Anatomy and Physiology 1871;16:233-40.

6. Anson BJ. Morris' Human Anatomy, $12^{\text {th }}$ edn. New York: McGrawHill. 1996.

7. Bryce TH. Note on a group of varieties of the pectoral sheet of muscle. Journal of Anatomy and Physiology 1899;34:75-8.

8. Macalister A. Additional observations on muscular anomalies in human anatomy (third series), with a catalogue of the principal muscular variations hitherto published. Transactions of the Royal Irish Academy 1871;25:117-30.

9. Chiba S. Suzuki T. Kasai T. A rare anomaly of the pectoralis major - the chondro-epitrochlearis. Okajimas Folia Anatomica Japonica 1983;60:175-86.

10. Voto SJ, Weiner Ds. The chondro-epitrochlearis muscle. Journal of Pediatric Orthopedics 1987;7:213-4.

11. Bergman RA. Doubled pectoralis quartus, axillary arch, chondroepitrochlearis, and the twist of the tendon of pectoralis major. Anatomischer Anzeiger 1991;173,23-6.

12. Bersu ET, Ramirex-Castro Jl. Anatomical analysis of the developmental defect of aneuploidy in man - the 18 trisomy syndrome: Anomalies of the head and neck. American Journal of Medical Genetics 1977;1:173-93. 